\title{
ORIGINAL
}

\section{MORTALIDAD ATRIBUIBLE AL ALCOHOL EN CATALUÑ̃: 1994}

\section{Miquel Alsedá Graells $(1,2)$ y Pere Godoy García $(1,2)$.}

(1) Sección de Epidemiología. Delegación Territorial del Departament de Sanitat i Seguretat Social de Lleida.

(2) Facultad de Medicina. Universidad de Lleida.

\section{RESUMEN}

Fundamento: El objetivo de este estudio es evaluar la contribución del consumo de alcohol a la mortalidad en Cataluña durante 1994, así como su contribución a las muertes prematuras.

Métodos: En este estudio se han utilizado los datos de las causas de mucrte en Cataluña durante 1994 del Servei d'Infor mació i Estudis de la Direcció General de Recursos Sanitaris del Departament de Sanitat i Seguretat Social de Catalunya. Las fracciones atribuibles al alcohol están basadas en las utilizadas en el estudio de la mortalidad relacionada con el alcohol en Estados Unidos en 1987.

Resultados: El 5.3\% de las muertes en Cataluna durante 1994 estuvieron relacionadas con el consumo de alcohol. Esta mortalidad fue del $6,5 \%$ entre los hombres y del $3,9 \%$ entre las mujeres. La categoría diagnóstica que más contribuyó al núInero de muertes fue la de las neoplasias malignas $(29,3 \%)$. El estudio de las muertes prematuras muestra que los accidentes no intencionales son la causa más importante $(52.3 \%$ ). En csta categoría diagnóstica los accidentes con vehículos a motor son los principales responsables de los años potenciales de vida perdidos (APVP). La media de APVP por cada muerte atribuible al alcohol hasta la edad de 65 fue de 7,5.

Conclusiones: Este estudio muestra la magnitud que tiene el alcohol como problema de salud pública en Cataluña.

Palahras clave: Alcohol. Mortalidad prematura. Años potenciales de vida perdidos (APVP). Accidentes de tráfico. Cataluña. Fracciones poblacionales atribuibles al alcohol. Investigación epidemiológica. Enfermedades crrebrovasculares. Cáncer.

\section{INTRODUCCIÓN}

El consumo excesivo de alcohol constituye uno de los principales problemas de salud pú-

Correspondencia:

Miquel Alsedà Graells,

C/ Camp de Mart. 39, 3, 2.

25004-Lleida.

Telf. 973-223935

\section{ABSTRACT \\ Alcohol Related Mortality in Catalonia: 1994}

Background: The objective of this study was to evaluate the contribution of the consumption of alcohol to mortality in Catalonia during 1994, as well as its importance in premature death.

Methods: This study used data on causes of death in Catalonia during 1994 from the «Servei D’Informació i Estudis de la Direcció General de Recursos Sanitaris del Departament de Sanitat i Seguretat Social de Catalunya» (Information and Study Service of the Directorate General for Health Care Resources. Catalonian Department of Health and Social Security). The figures attributable to alcohol are based on those used in the study on alcohol-related mortality in the United States in 1987

Results: In $19945.3 \%$ of deaths in Catalonia were related to the consumption of alcohol. The mortality rate was $6.5 \%$ for men, and $3.9 \%$ for women. The diagnostic category which most contributed to the number of deaths was that of malignant neoplasias $(29.3 \%)$. The study of premature deaths show that accidents were the major cause $(52.3 \%)$. In this diagnostic category car accidents were the main cause of potential years of life lost (PYLL). The average PYI I. for each death attributable to alcohol up to the age of 65 was 7.5 .

Conclusions: This study shows the scale of alcohol as a public health problem in Catalonia.

Key words: Alcohol-induced mortality. Premature death from alcohol. Catalonia (Spain).

blica en los países desarrollados. Esta preocupación aparece reflejada en el Plan de Salud de Cataluña en el cual se fija como objetivo general de salud número 12 , la reducción en un $15 \%$ de la mortalidad atribuible al alcoholismo en el período 1993-2000'.

Diferentes estudios epidemiológicos han puesto de manifiesto la relación entre un elevado consumo de alcohol y un incremento 
en el riesgo de mortalidad ${ }^{2-5}$. Algunas de estas causas de muerte son enfermedades directa o indirectamente producidas por el efecto tóxico del alcohol y las restantes lo son por alteraciones de la conducta o por circunstancias que están favorecidas por el consumo de bebidas alcohólicas.

La contribución del consumo excesivo de alcohol en la mortalidad ha sido evaluada en diversas publicaciones ${ }^{6,7}$. En todos los casos, estas estimaciones están basadas en revisiones de la literatura médica e intentan cuantificar la contribución del alcohol en diversas causas de muerte. Entre estas revisiones, la más reciente es la utilizada en el cálculo de la mortalidad atribuible al alcohol en los Estados Unidos, que fue del 4,9\% en el año $1987^{\circ}$.

El objetivo del estudio es la estimación de la mortalidad atribuible al alcohol y los años potenciales de vida perdidos (APVP) en Cataluña durante el año 1994.

\section{MATERIAL Y MÉTODOS}

El conjunto de las causas de muerte incluidas en el estudio han sido aquellas relacionadas con el consumo excesivo de alcohol. Los estimadores de impacto del alcohol utilizados han sido las llamadas fracciones poblacionales atribuibles al alcohol

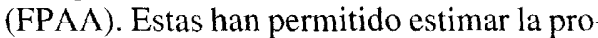
porción de muertes que en cada una de las causas estudiadas están relacionadas con el consumo de alcohol. Las causas de muerte y las FPAA que se han utilizado están basadas en las expuestas en el estudio de la mortalidad atribuible al alcohol en Estados Unidos el año $1987^{\circ}$.

Los datos de mortalidad de Cataluña han sido facilitados por el Servei d'Informació i Estudis de la Direcció General de Recursos Sanitaris del Departament de Sanitat i Seguretat Social de la Generalitat de Catalunya. Se ha dispuesto de los datos de 1994 por categoría diagnóstica según la Clasificación Internacional de Enfermedades, Novena
Revisión, Modificación Clínica (CIE-9MC), desagregados por grupos de edad en períodos de 5 años y sexo. Se utilizaron los datos de 1994 por ser el último año del cual se pudo disponer de información sobre la mortalidad en el momento de realizar el estudio.

La mortalidad atribuible al alcohol se ha calculado multiplicando el número de muertes en cada categoría diagnóstica por las correspondientes FPAA por sexo y período de 5 años de edad. Hay que tener en cuenta que algunas FPAA se han aplicado solamente a las muertes de personas por encima de cierta edad y para dos causas de muerte las FPAA son específicas por sexo.

Para valorar el impacto del alcohol en las muertes que tienen lugar en las edades más jóvenes, hemos calculado los APVP. En este estudio hemos estimado los APVP hasta los 65 años que coincide con la finalización de la cdad laboral en nuestro país. Para cada categoría diagnóstica los APVP han sido calculados multiplicando el número de muertes por las correspondientes FPAA y por los APVP hasta los 65 años por sexo y período de 5 años de edad.

\section{RESULTADOS}

El número total de muertes atribuibles al consumo excesivo de alcohol según el modelo aplicado es de 2.764. Estas representan el $5,3 \%$ de todas las muertes (52.205) producidas en Cataluña durante el año 1994.

Del total de muertes atribuibles al alcohol, el $65,1 \%$ corresponde a hombres (1.799), y el $34,9 \%$ a mujeres (965). Estas cifras representan el $6,5 \%$ y el $3,9 \%$ de todas las muertes producidas en hombres (27.604) y mujeres (24.601), respectivamente.

El $15,8 \%$ de las muertes atribuibles al alcohol corresponden a otras cirrosis hepáticas (436), el $15,4 \%$ a enfermedades cerebrovasculares (426), el 10,7\% a accidentes de vehí- 
culos a motor (295), el 7,5\% a cáncer de esófago (206) y el 7,2\% a cáncer de estómago (200). Estos cinco diagnósticos representan el $56,6 \%$ de las muertes atribuibles al alcohol (tabla 1).

Respecto a los grupos diagnósticos, el $29,3 \%$ de las muertes atribuibles al alcohol corresponden a neoplasias malignas (810), el $22.8 \%$ a enfermedades digestivas (631), el $21,1 \%$ a accidentes no intencionales (584) y el $16,2 \%$ a enfermedades cardiovasculares (448). El conjunto de estos cuatro grandes grupos diagnósticos representa, el $89,4 \%$ de las muertes atribuibles al alcohol (tabla 2).

Se observan diferencias en la distribución de las categorías diagnósticas por sexos. $\mathrm{La}$ razón hombre/mujer presenta valores superiores a las dos unidades en los transtornos mentales. las neoplasias malignas, los accidentes intencionales y los no intencionales. Esta razón es inferior a la unidad en las enfermedades cardiovasculares y en las otras enfermedades relacionadas con el alcohol (tabla 2).

El número total de APVP es de 20.763. De estos, el $80,9 \%$ corresponden a los hombres (16.801) y el $19.1 \%$ a las mujeres (3.962). Por diagnósticos específicos, el $36,6 \%$ de APVP corresponden a accidentes de vehículos a motor $(7.600)$. Por grandes grupos diagnósticos, el 52,3\% de APVP corresponden a accidentes no intencionales (10.854). En la distribución por sexos, los accidentes no intencionales ocupan el primer lugar de APVP en los dos sexos, en los hombres son el $51,8 \%(8.707)$ y en las mujeres el 54,2\% (2.147) (tabla 3).

La media de APVP por cada muerte atribuible al alcohol es de 7,5. Por sexos, en los hombres es de 9,3 y en las mujeres de 4,1 . La categoría diagnóstica que presenta una media más alta son los accidentes no intencionales en los hombres 21.8 y los accidentes intencionales en las mujeres 14,5 (tabla 4).

\section{DISCUSIÓN}

El presente estudio muestra la importancia del consumo excesivo de alcohol en la mortalidad registrada en Cataluña. Según el modelo utilizado, el 5,3\% de todas las muertes producidas en Cataluña durante el año 1994 son atribuibles al alcohol. Este porcentaje es superior al $4 \%$ citado en el Plan de Salud de Cataluña ${ }^{1}$. El porcentaje de nuestro estudio es también superior al 4,08\% obtenido en el análisis de la mortalidad atribuible al alcohol en España el año 1985, si bien en este estudio solamente se consideran las principales causas de muerte atribuibles al alcohol ${ }^{9}$. En dos estudios realizados en España, el primero para estudiar la mortalidad del año 1986 y el segundo para estudiar la mortalidad en el período 1981-1990, en los cuales se ha utilizado la misma metodología, los porcentajes son de $6,1 \%$ y $6,3 \%$ (media del período) respectivamente ${ }^{10-11}$. Este hecho coincide con los estudios de prevalencia de consumo en la población general que encuentran un porcentaje superior de consumo de riesgo en el global de España respecto a Cataluña ${ }^{12-14}$.

El riesgo de morir por causas relacionadas con el consumo de alcohol es casi el doble en los hombres respecto a las mujeres. Este riesgo se mantiene más o menos constante tanto en los estudios americanos ${ }^{8.15 .16}$ como en los realizados en España ${ }^{10-11}$. Cuando se analiza la aportación por sexos a los APVP, ésta es 4,2 veces superior en los hombres respecto a las mujeres. Este hecho es debido a que los hombres presentan una media de APVP por cada muerte atribuible al alcohol 2,3 veces superior respecto a las mujeres. En el estudio americano de referencia ${ }^{8}$, la aportación de los hombres a los APVP es del triple respecto a las mujeres. Esto es debido a que la media de APVP por cada muerte atribuible en los hombres es 1,5 veces superior a la de las mujeres.

Respecto al global de las categorías diagnósticas, es necesario remarcar los grandes cambios que se producen en los pesos por- 
Tabla 1

Fracciones poblacionales atribuibles al alcohol (FPAA), mortalidad total y mortalidad atribuible al alcohol (MAA), por sexo y diagnóstico. Cataluña 1994

\begin{tabular}{|c|c|c|c|c|c|c|}
\hline \multirow[b]{2}{*}{ Diagnóstico $(C I E-9-M C)$} & \multicolumn{2}{|c|}{ Edad } & \multicolumn{2}{|c|}{ Hombre } & \multicolumn{2}{|c|}{ Mijer } \\
\hline & $F P A A$ & $a \tilde{o} o s^{a}$ & $\begin{array}{c}\text { Núm. } \\
\text { muertes }\end{array}$ & $M A A$ & $\begin{array}{c}\text { Núm. } \\
\text { muertes }\end{array}$ & $M A A$ \\
\hline \multicolumn{7}{|l|}{ Neoplasias malignas } \\
\hline Cáncer de labio/cavidad oral/faringe (140-149) & $0,50^{\mathrm{b}}$ & $\geq 35$ & 300 & 150 & 50 & 20 \\
\hline Cáncer de esófago (150) & 0,75 & $\geq 35$ & 241 & 181 & 33 & 25 \\
\hline Cáncer de estómago (151) & 0,20 & $\geq 35$ & 588 & 118 & 410 & 82 \\
\hline Cáncer de hígado/conductos biliares intrahepáticos (155) & 0,15 & $\geq 35$ & 447 & 67 & 247 & 37 \\
\hline Cáncer de laringe (161) & $0,50^{\mathrm{b}}$ & $\geq 35$ & 251 & 126 & 11 & 4 \\
\hline \multicolumn{7}{|l|}{ Transtornos mentales } \\
\hline Psicosis alcohólica (291) & 1,00 & $\geq 15$ & 4 & 4 & 0 & 0 \\
\hline Síndrome de dependencia alcohólica (303) & 1,00 & $\geq 15$ & 18 & 18 & 3 & 3 \\
\hline Abuso de alcohol (305.0) & 1,00 & $\geq 15$ & 1 & 1 & 0 & 0 \\
\hline \multicolumn{7}{|l|}{ Enfermedades cardiovasculares } \\
\hline Hipertensión esencial (401) & 0,08 & $\geq 35$ & 75 & 6 & 149 & 12 \\
\hline Cardiomiopatía alcohólica (425.5) & 1,00 & $\geq 15$ & 2 & 2 & 2 & 2 \\
\hline Enfermedades cerebrovasculares $(430-438)$ & 0,07 & $\geq 35$ & 2503 & 175 & 3588 & 251 \\
\hline \multicolumn{7}{|l|}{ Enfermedades respiratorias } \\
\hline Tuberculosis respiratoria (011-012) & 0,25 & $\geq 35$ & 48 & 12 & 18 & 5 \\
\hline Neumonía y gripe $(480-487)$ & 0,05 & $\geq 35$ & 407 & 20 & 382 & 19 \\
\hline \multicolumn{7}{|l|}{ Enfermedades digestivas } \\
\hline Enfermedades del esófago/estómago/duodeno (530-5.37) & 0,10 & $\geq 35$ & 112 & 11 & 91 & 9 \\
\hline Gastritis alcoholica (535.3) & 1,00 & $\geq 15$ & 0 & 0 & 0 & 0 \\
\hline Degeneración grasa alcohólica del hígado (571.0) & 1,00 & $\geq 15$ & 0 & 0 & 0 & 0 \\
\hline Hepatitis alcohólica aguda (571.1) & 1,00 & $\geq 15$ & 7 & 7 & 4 & 4 \\
\hline Cirrosis alcohólica (571.2) & 1,00 & $\geq 15$ & 59 & 59 & 19 & 19 \\
\hline Lesión alcohólica inespecificada del hígado (571.3) & 1,00 & $\geq 15$ & 27 & 27 & 6 & 6 \\
\hline Otras cirrosis del hígado $(571.5,571.6)$ & 0,50 & $\geq 35$ & 513 & 257 & 358 & 179 \\
\hline Pancreatitis aguda $(577.0)$ & 0,42 & $\geq 35$ & 56 & 24 & 65 & 27 \\
\hline Pancreatitis crónica (577.1) & 0,60 & $\geq 35$ & 2 & 1 & 1 & 1 \\
\hline \multicolumn{7}{|l|}{ Accidentes no intencionales } \\
\hline Accidentes de vehículos a motor (E810-E825) & 0,42 & $>0$ & 541 & 227 & 161 & 68 \\
\hline Otros accidentes de vehículos viarios (E826-E829) & 0.20 & $>0$ & 8 & 2 & 0 & 0 \\
\hline Accidentes de transporte acuático (E830-E838) & 0,20 & $>0$ & 1 & 0 & 0 & 0 \\
\hline Accidentes de transporte aéreo y espacial (E840-E845) & 0,16 & $>0$ & 3 & 0 & 0 & 0 \\
\hline Envenenamientos por alcohol (E860.0, E860.1) & 1,00 & $\geq 15$ & 1 & 1 & 0 & 0 \\
\hline Caídas accidentales (E880-E888) & 0,35 & $\geq 15$ & 155 & 54 & 184 & 64 \\
\hline Accidentes causados por el fuego (E890-E899) & 0,45 & $>0$ & 23 & 10 & 15 & 7 \\
\hline Accidentes durante el baño (E910) & 0,38 & $>0$ & 62 & 24 & 17 & 6 \\
\hline Otros accidentes $^{c}$ & 0,25 & $\geq 15$ & 327 & 82 & 157 & 39 \\
\hline \multicolumn{7}{|l|}{ Accidentes intencionales } \\
\hline Suicidios (E950-E959) & 0,28 & $\geq 15$ & 342 & 96 & 121 & 34 \\
\hline Homicidios (E960-E969) & 0,46 & $\geq 15$ & 26 & 12 & 12 & 6 \\
\hline \multicolumn{7}{|l|}{ Enfermedades metabólicas } \\
\hline Diabctes mellitus (250) & 0,05 & $\geq 35$ & 472 & 24 & 726 & 36 \\
\hline \multicolumn{7}{|l|}{ Otras enfermedades relacionadas con el alcohol } \\
\hline Polineuropatía alcohólica (357.5) & 1,00 & $\geq 15$ & 1 & 1 & 0 & 0 \\
\hline Nivel excesivo de alcohol en sangre (790.3) & 1,00 & $\geq 15$ & 0 & 0 & 0 & 0 \\
\hline Total & & & 7.632 & 1.799 & 6.830 & 965 \\
\hline
\end{tabular}

a Las muertes que han tenido lugar antes de esta edad no han sido incluidas en los cálculos.

" La FPAA es de 0,40 para las mujeres.

' Los códigos CIE-9-MC incluidos son E800-E807. E900-E909, E911, E916-E928 E980-E989. 
Tabla 2

Mortalidad atribuible al alcohol y razón hombre/mujer, por sexo y categoría diagnóstica. Cataluña 1994

\begin{tabular}{|c|c|c|c|c|c|c|c|}
\hline \multirow{2}{*}{ Categoría diagnóstica } & \multicolumn{6}{|c|}{ Número de muertes } & \multirow{2}{*}{$\begin{array}{c}\text { Razón } \\
\text { Hombre/ } \\
\text { Mujer }\end{array}$} \\
\hline & Hombre & $(\%)$ & Mujer & $(\%)$ & Total & $(\%)$ & \\
\hline Neoplasias malignas & 642 & $(35,7)$ & 168 & $(17,4)$ & 810 & $(29,3)$ & 3,8 \\
\hline Transtornos mentales & 23 & $(1,3)$ & 3 & $(0,3)$ & 26 & $(0,9)$ & 7,7 \\
\hline Enfermedades cardiovasculares & 183 & $(10,2)$ & 265 & $(27,5)$ & 448 & $(16,2)$ & 0.7 \\
\hline Enfermedades respiratorias & 32 & $(1,8)$ & 24 & $(2,5)$ & 56 & $(2,0)$ & 1.3 \\
\hline Enfermedades digestivas & 386 & $(21,5)$ & 245 & $(25,4)$ & 631 & $(22,8)$ & 1,6 \\
\hline Accidentes no intencionales & 400 & $(22,2)$ & 184 & $(19,1)$ & 584 & $(21,1)$ & 2,2 \\
\hline Accidentes intencionales & 108 & $(6,0)$ & 40 & $(4,1)$ & 148 & $(5,4)$ & 2,7 \\
\hline Otras enfermedades relacionadas con el alcohol ${ }^{\mathrm{a}}$ & 25 & $(1,4)$ & 36 & $(3,7)$ & 61 & $(2,2)$ & 0,7 \\
\hline Total & 1.799 & & 965 & & 2.764 & & \\
\hline
\end{tabular}

"Incluye: diabetes meilitus. polineuropatía alcohólica y nivel excesivn de alcotiol en sangre.

Tabla 3

Años potenciales de vida perdidos (APVP) atribuibles al alcohol, por sexo y categoría diagnóstica. Cataluña 1994

\begin{tabular}{|c|c|c|c|c|c|c|}
\hline \multirow{2}{*}{ Categoría diagnóstica } & \multicolumn{6}{|c|}{ APVP hasta los 65 años } \\
\hline & Hombre & $(\%)$ & Mujer & $(\%)$ & Total & $(\%)$ \\
\hline Neoplasias malignas & 3.048 & $(18,1)$ & 307 & $(7,7)$ & 3.355 & $(16,2)$ \\
\hline Transtornos mentales & 242 & $(1,4)$ & 36 & $(0,9)$ & 278 & $(1,3)$ \\
\hline Enfermedades cardiovasculares & 199 & $(1,2)$ & 110 & $(2,8)$ & 309 & $(1,5)$ \\
\hline Enfermedades respiratorias & 124 & $(0,7)$ & 5 & $(0,1)$ & 129 & $(0,6)$ \\
\hline Enfermedades digestivas & 2.453 & $(14,6)$ & 763 & $(19,3)$ & 3.216 & $(15,5)$ \\
\hline Accidentes no intencionales & 8.707 & $(51,8)$ & 2.147 & $(54,2)$ & 10.854 & $(52,3)$ \\
\hline Accidentes intencionales & 1.997 & $(11,9)$ & 579 & $(14,6)$ & 2.576 & $(12,4)$ \\
\hline Otras enfermedades relacionadas con el alcohol ${ }^{\mathrm{a}}$ & 31 & $(0,2)$ & 15 & $(0,4)$ & 46 & $(0,2)$ \\
\hline Total & 16.801 & & 3.962 & & 20.763 & \\
\hline
\end{tabular}

Incluye: diabetes mellitus, polineuropatía alcohólica y nivel excesivo de alcohol en sangrc.

Tabla 4

Media de años potenciales de vida perdidos (APVP) atribuibles al alcohol, por sexo y categoría diagnóstica. Cataluña 1994

\begin{tabular}{|c|c|c|c|}
\hline \multirow{2}{*}{ Categoría diagnóstica } & \multicolumn{3}{|c|}{ Media de APVP hasta los 65 años } \\
\hline & Hombre & Mujer & Total \\
\hline Neoplasias malignas & 4.7 & 1,8 & 4,1 \\
\hline Transtornos mentales & 10,5 & 12,0 & 10,7 \\
\hline Enfermedades cardiovasculares & 1,1 & 0,4 & 0,7 \\
\hline Enfermedades respiratorias & 3,9 & 0,2 & 2.3 \\
\hline Enfermedades digestivas & 6,4 & 3,1 & 5,1 \\
\hline Accidentes no intencionales & 21,8 & 11,7 & 18.6 \\
\hline Accidentes intencionales & 18,5 & 14,5 & 17.4 \\
\hline Otras enfermedades relacionadas con el alcohol ${ }^{a}$ & 1,2 & 0,4 & 0.8 \\
\hline Total & 9,3 & 4,1 & 7,5 \\
\hline
\end{tabular}

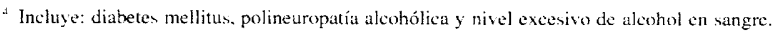


centuales de algunas de ellas, según se utilice como criterio de referencia el número de muertes o los APVP. Estos cambios nos llevan a concluir que los accidentes no intencionales y los intencionales causan una mayor mortalidad atribuible al alcohol en edades jóvenes, mientras que las enfermedades cardiovasculares y las neoplasias malignas causan mayor mortalidad en edades más avanzadas.

En relación a los diagnósticos específicos, es necesario tener presente la importancia de los accidentes de vehículo a motor que representan el $36,6 \%$ de los APVP. Esta relación entre el alcohol y los accidentes en general, y los de vehículo a motor en particular se encuentra bien documentada en nuestro entorno ${ }^{17-19}$.

La media de 7,5 APVP por cada muerte atribuible al alcohol es muy similar a la de 7,3 del estudio español ${ }^{10}$. Pero éstas son aproximadamente la mitad de la media de 14,6 expucsta en el artículo americano de referencia ${ }^{8}$. Este hecho viene determinado en gran parte, por un mayor peso de la mortalidad producida por las lesiones no intencionales $28,7 \%$ e intencionales $16,8 \%$ en el estudio americano ${ }^{8}$ comparado con el $21,1 \%$ y $5,4 \%$ respectivamente, de nuestro estudio.

La metodología utilizada en el presente trabajo no está exenta de limitaciones. En primer lugar, es necesario que en los datos de mortalidad, el número de muertes por los diferentes diagnósticos específicos se ajuste a la realidad. Se han descrito errores en la certificación de la causa de la muerte que pueden infravalorar la influencia del alcohol en la mortalidad ${ }^{20.21}$. En segundo lugar, las FPAA dependen de la exposición al alcohol y de la magnitud del efecto (riesgo relativo). La discusión detallada de las dificultades metodológicas en la valoración de los parámetros relacionados con cada uno de los dos factores anteriores ya aparece bien descrita en la literatura médica ${ }^{22}$. También es necesario añadir que la utilización de una única FPAA no desagregada por edad y sexo es problemática. Por otro lado, la aplicación en Cataluña de las FPAA utilizadas en los Estados Unidos es otra limitación de este trabajo. En este caso, debido a la falta de estudios al respecto en Cataluña, hemos asumido que no existen grandes diferencias con las FPAA expuestas en el estudio americano de referencia ${ }^{8}$.

A pesar de las dificultades metodológicas citadas anteriormente, el presente estudio nos permite una aproximación al impacto del consumo excesivo de alcohol en la salud pública de nuestra comunidad. Así como también nos permite disponer de una herramienta útil para monitorizar y evaluar el efecto de las intervenciones sanitarias.

Estos resultados confirman la importancia de la contribución del consumo excesivo de alcohol en la mortalidad y en los APVP en Cataluña, así como la necesidad de actuaciones preventivas de acuerdo con los criterios fijados en nuestro entorno ${ }^{23.24}$.

\section{BIBLIOGRAFÍA}

1. Departament de Sanitat i Seguretat Social. Plan de Salud de Cataluña 1993-1995.1. ${ }^{a}$ ed. Barcelona: Departament de Sanitat i Seguretat Social; 1993 , pp. 130-132, 216-219.

2. Smith $R$. The relation betwen consumption and damage. Br Med J 1981; 283: 895-898.

3. Gordon T, Doyle JT. Drinking and mortality. Am J Epidemiol 1987; 125: 263-270.

4. Williams GD, Grant BF, Stinson FS, Zobeck TS, Aitken SS, Noble J. Trends in alcohol-related morbidity and mortality. Public Health Rep 1988; 103(6): 592-597.

5. Duffy JC. Alcohol consumption and all-causc mortality. Int J Epidemiol 1995; 24: 100-105.

6. Parker D, Shultz J, Gertz L, Berkelman R, Remington $P$. The social and economic cost of alcohol abuse in Minnesota. Am J Public Health 1987; 77: $982-986$

7. Mcdonnell R, Maynard A. Estimation of life years lost from alcohol-related premature death. Alcohol Alcoholism 1985; 20: 435-443. 
8. Shultz JM, Rice DP, Parker DL. Alcohol-related mortality and years of potential life lost-United States, 1987. MMWR 1990; 39: 173-178.

9. Salleras L, Bach L. Alcohol y Salud. En: Piédrola G, del Rey J, Domínguez M, Cortina P, Gálvez R, Sierra A, et al. Medicina Preventiva y Salud Pública. 9." edición. Barcelona: Masson-Salvat; 1992. pp. 1.278-1.293.

10. Yañez JL, Del Rio MC, Alvarez. FJ. Alcohol-relatcd mortality in Spain. Alcohol Clin Exp Res $1993 ; 17: 253-255$.

11. Prada C, Del Río MC, Yáñez JL, Álvarez FJ. Mortalidad relacionada con el consumo de alcohol en España: 1981-1990. Gac Sanit 1996; 10: 161-168.

12. Majó X, Ollé JM, Suelves JM, Torralba L1, Colom J. El consum i les actituds de la població catalana respecte a l'alcohol i les drogues socialment no integrades. Enquesta 1990. Salut Catalunya 1994; 8: 115-120.

13. Gil López E, Jiménez García Pascual R, Pérez Andrés C y Vargas Marcos F. Estudio de los estilos de vida de la población adulta española. Madrid: Ministerio de Sanidad y Consurno; 1990.

14. Cruz Roja Española y Ministerio de Trabajo. El consumo de drogas en España. Cuadernos de toxicomanías (núm. 14). Madrid: Cruz Roja Española; 1985.

15. Sutocky JW, Shultz JM, Kizer KW. Alcohol-related mortality in California, 1980 to 1989 . Am J Public Health 1993; 83: 817-823.
16. Akgulian NA, Moss ME, Remington PL, Anderson HA, Shultz JM. Alcohol-related disease impact-Wisconsin, 1988. MMWR 1990; 39: 178 $180,185-187$.

17. Pares A, Caballeira J, Rodamilans M, Urbano A, Bach L, Rodés J. Consumo de alcohol y accidentes en Barcelona. Estudio epidemiológico. Med Clin (Barc) 1988; 90: 759-762.

18. Salleras L. Alcohol y accidentes. Med Clin (Barc) 1988; 90: 775-778.

19. Cía MT, Arévalo JM, Ardanaz E, Barricarte A, Chueca P, Serrano S. Nivel de alcohol y riesgo de lesión por accidente de tráfico en Tudela (Navarra). Gac Sanit 1996; 10: 55-61.

20. Pollock DA, Boyle CA, DeStefano F, Moyer LA, Kirk ML. Underreporting of alcohol-related mortality on death certificates of young US Army veterans. JAMA 1987; 258: 345-348.

21. Romelsjo A, Karlsson G, Henningsohn L, Jakobsson SW. The prevalence of alcohol-related mortality in both sexes: variation betwen indicators, Stockholm, 1987. Am J Public Health 1993; 83(6): 838-844.

22. González J, Rodríguez F, Martín I, Banegas JR, Villar F. Muertes atribuibles al consumo de tabaco en España. Med Clin (Barc) 1989; 92: 15-18.

23. Bruguera M, Gual A, Salleras L, Rodés J. Cribado del consumo excesivo de alcohol. Med Clin (Barc) 1994; 102 Supl 1: 85-92.

24. Robledo T, Álvarez FJ. Plan Europeo de actuación sobre el consumo de alcohol: una mirada hacia el futuro. Med Clin (Barc) 1996; 106: 581-582. 